\title{
Catecholamine Storage Vesicles: Role of Core Protein Genetic Polymorphisms in Hypertension
}

\author{
Kuixing Zhang • Yuqing Chen • Gen Wen • Manjula Mahata • Fangwen Rao • \\ Maple M. Fung • Sucheta Vaingankar • Nilima Biswas • Jiaur R. Gayen • \\ Ryan S. Friese • Sushil K. Mahata • Bruce A. Hamilton • Daniel T. O'Connor
}

Published online: 24 November 2010

(C) The Author(s) 2010. This article is published with open access at Springerlink.com

\begin{abstract}
Hypertension is a complex trait with deranged autonomic control of the circulation. The sympathoadrenal system exerts minute-to-minute control over cardiac output and vascular tone. Catecholamine storage vesicles (or chromaffin granules) of the adrenal medulla contain remarkably high concentrations of chromogranins/secretogranins (or "granins"), catecholamines, neuropeptide Y, adenosine triphosphate (ATP), and $\mathrm{Ca}^{2+}$. Within secretory granules, granins are co-stored with catecholamine neurotransmitters and co-released upon stimulation of the regulated secretory pathway. The principal granin family members, chromogranin A $(C H G A)$, chromogranin $\mathrm{B}$ $(C H G B)$, and secretogranin II (SCG2), may have evolved from shared ancestral exons by gene duplication. This article reviews human genetic variation at loci encoding the major granins and probes the effects of such polymorphisms on blood pressure, using twin pairs to probe
\end{abstract}

K. Zhang $\cdot$ Y. Chen $\cdot$ G. Wen $\cdot$ M. Mahata $\cdot$ F. Rao $\cdot$ M. M. Fung $\cdot$

S. Vaingankar $\cdot$ N. Biswas $\cdot$ J. R. Gayen $\cdot$ R. S. Friese $\cdot$

S. K. Mahata • B. A. Hamilton • D. T. O'Connor $(\bowtie)$

Department of Medicine and Institute for Genomic Medicine

(IGM), University of California at San Diego School of Medicine,

9500 Gilman Drive,

La Jolla, CA 92093-0838, USA

e-mail: doconnor@ucsd.edu

D. T. O'Connor

Department of Pharmacology,

University of California at San Diego,

San Diego, CA, USA

M. M. Fung $\cdot$ S. K. Mahata $\cdot$ D. T. O'Connor

VA San Diego Healthcare System,

San Diego, CA, USA heritability and individuals with the most extreme blood pressure values in the population to study hypertension.

Keywords Genetics · Hypertension - Catecholamine . Granins · Blood pressure · CHGA · CHGB · SCG2 . Heritability

\section{Introduction: The Sympathoadrenal System and Granins}

The sympathoadrenal system exerts minute-to-minute control over cardiac output and vascular tone. Genes governing catecholaminergic processes may play a role in the development of hypertension. Catecholamine storage vesicles (or chromaffin granules) of the adrenal medulla (Fig. 1) contain remarkably high concentrations of chromogranins/secretogranins ("granins"), neuropeptide Y (NPY), catecholamines, adenosine triphosphate (ATP), and $\mathrm{Ca}^{2+}$. Within the vesicle core, granins bind catecholamines and ATP, to stabilize the core osmotically $[1,2]$. When the major granin, chromogranin A (CHGA), is depleted by gene targeting, adrenal chromaffin granules are reduced in number, size, and electron density, and transmitter secretion from the regulated pathway is disrupted [3]. Granins also bind to the inner aspect of the vesicle membrane, where they may influence the release of calcium from secretory granules to the cytosolic exocytotic machinery through the inositol 1,4,5-trisphosphate receptor/ $\mathrm{Ca}^{2+}$ channel [4].

$C H G A$, chromogranin B $(C H G B)$, and secretogranin II $(S C G 2)$ are paralogous genes that likely arose by gene duplication within the vertebrate lineage [5], suggesting closely related cellular and physiological functions. Heterologous expression of any one of the three "granins" is sufficient to induce secretory granules and regulated 


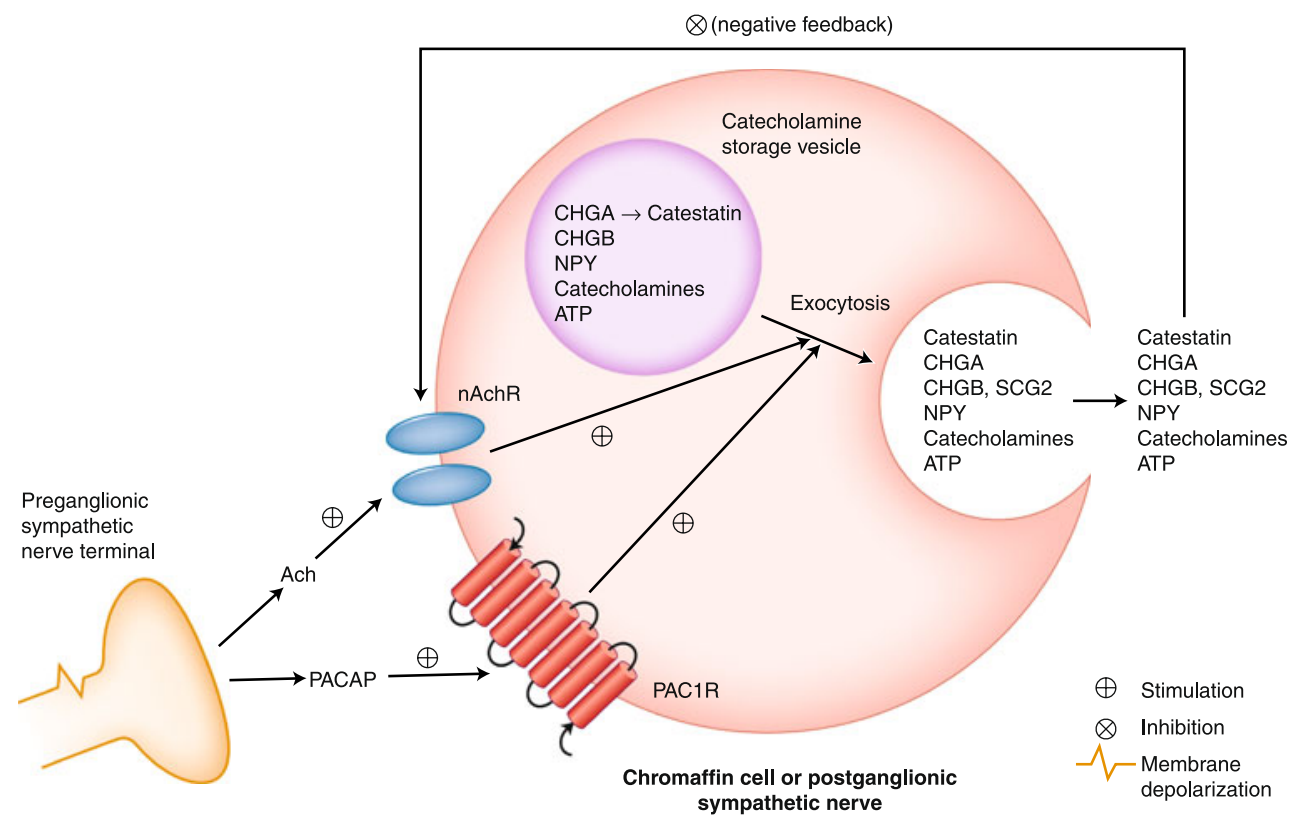

Fig. 1 Intracellular and extracellular chromogranin/secretogranin actions. Chromaffin granule transmitter storage and exocytotic release are depicted, with secretion in response to the preganglionic transmitters acetylcholine (Ach) or pituitary adenylyl cyclase activating polypeptide (PACAP). Catestatin, the endogenous nicotinic cholinergic antagonist (ganglionic-blocking) peptide fragment of CHGA, inhibits the principal physiological trigger of chromaffin cell secretory stimulation: the nicotinic cholinergic pathway. ATP-adenosine triphosphate; CHGA - chromogranin A; CHGB - chromogranin B; nAchR - nicotinic cholinergic receptor; NPY - neuropeptide Y; PAC1R-PACAP type 1 receptor; SCG2 - secretogranin II. (Adapted from Mahapatra et al. [1]) secretion in cells that otherwise lack both [6-8], and depletion of endogenous CHGA impairs formation of chromaffin granules in PC12 chromaffin cells [3]. Within secretory granules, granins are co-stored with catecholamine neurotransmitters and are co-released upon stimulation of regulated secretion. Information on chromosomal positions, genomic structure, cDNA, and proteins encoded by the three genes is displayed in Table 1.

Proteolytic cleavage of granins at dibasic sites generates an array of bioactive peptides within secretory vesicles. In aggregate, these peptides have physiological effects on cardiovascular, endocrine, metabolic, and immune systems through both direct and indirect participation of the sympathetic nervous system [9]. CHGA is a pro-hormone that gives rise to at least four biologically active fragments: the dysglycemic peptide pancreastatin $[10,11]$; the antimicrobial peptide chromacin [12]; the amino-terminal vasodilator vasostatin [13]; and the catecholamine release inhibitor catestatin [14, 15]. As a pro-protein, CHGB is cleaved to biologically active fragments including "GAWK" $[16,17]$ (bovine $\mathrm{Chgb}_{398-464}$, human $\mathrm{CHGB}_{420-493}$ ), “CCB” [16] (human $\mathrm{CHGB}_{597-656}$ ), "PE-11" [18] (rat $\mathrm{Chgb}_{532-542}$, human $\mathrm{CHGB}_{536-545}$ ), and the endogenous antimicrobial/bacteriolytic peptides chrombacin [19] (bovine Chgb 564-626, human $\mathrm{CHGB}_{597-657)}$ ) and secretolytin [20] (bovine $\mathrm{Chgb}_{614-626}$, human $\mathrm{CHGB}_{647-657)}$ ). SCG2 is the precursor of the 33-amino acid peptide secretoneurin (SN, human SCG2 165-187), which modulates neurotransmission and inflammatory responses and may be involved in neuronal differentiation [21].

This review first considers the cellular and physiological mechanisms of granin action that relate to sympathoadrenal regulation, and then reviews recent findings on associations

Table 1 Genomic structure of classic/major human chromogranin/secretogranins: CHGA, CHGB, and SCG2

\begin{tabular}{lllcccccc}
\hline Gene & Chromosome & Chromosomal position & Genomic size (bp) & cDNA (bp) & Exons & Amino acids & Signal peptide & Molecular weight (Da) \\
\hline CHGA & $14 \mathrm{q} 32$ & $93389445-93401636$ & 12,192 & 2,079 & 8 & 457 & $1-18$ & 48,918 \\
CHGB & $20 \mathrm{p} 12.3$ & $5891974-5906003$ & 14,030 & 2,666 & 5 & 677 & $1-20$ & 76,326 \\
$S C G 2$ & $2 \mathrm{q} 36.1$ & $224461660-224467121$ & 5,462 & 2,490 & 2 & 617 & $1-27$ & 70,810 \\
\hline
\end{tabular}

$B p$ base pairs; $D a$ Daltons 
of genetic variants in these genes with blood pressure and intermediate phenotypes that may underlie susceptibility to hypertension.

\section{Granin Secretion and Hypertension}

\section{CHGA}

Basal plasma CHGA concentration is correlated with sympathetic tone [22, 23], and studies in twins indicate that the basal level is highly heritable [24]. As compared with agematched normotensive controls, patients with essential hypertension have increased plasma CHGA and an increased release of stored CHGA in response to adrenal medullary stimulation by insulin-evoked hypoglycemia [24]. The dysglycemic CHGA fragment pancreastatin is also elevated in patients with essential hypertension with or without obesity $[25,26]$; its actions may therefore contribute to the insulin resistance that often accompanies the condition. Catestatin, the CHGA-derived peptide that inhibits catecholamine release, is decreased in patients with essential hypertension and even in normotensive subjects with a family history of hypertension [27]. Moreover, people with such a family history have increased epinephrine secretion in addition to diminished catestatin, suggesting an inhibitory effect of catestatin on chromaffin cells in vivo. Low catestatin levels also predict augmented adrenergic response to stressors, suggesting that reduction in catestatin increases risk of hypertension [27]. The catestatin Gly364Ser variant causes profound changes in human autonomic activity, both parasympathetic and sympathetic, and seems to reduce risk of developing hypertension, especially in men. A model for catestatin action in the baroreceptor center of the nucleus of the tractus solitarius accounts for these actions [28]. Consistent with these findings in humans, expression of the Chga gene was observed to be significantly higher in adrenal glands of rat and mouse models of genetic hypertension [2931], supporting the phenotypic association between elevated CHGA and essential hypertension.

\section{CHGB}

Expression of CHGB may mark the action of still poorly characterized trans-quantitative trait loci influencing exocytotic sympathoadrenal activity $[32,33]$. Like CHGA, CHGB may be differentially expressed in rodent models of genetic hypertension [29, 30] and acquired hypertension [34], further suggesting augmented sympathoadrenal activity in the pathogenesis of these syndromes. CHGB may thereby give rise to early, pathogenic "intermediate phenotypes" [35] for exploration of sympathoadrenal activity in human essential hypertension.

\section{SCG2}

The secretoneurin peptide derived from SCG2 (human SCG2 $165-187$ ) stimulates migration and proliferation of vascular smooth muscle cells [21] and acts as an endothelial cytokine to promote angiogenesis and vasculogenesis [30, 36], providing a direct link to vascular development and remodeling and a potential model for its involvement in autonomic phenotypes.

\section{Population Samples and Traits for Genetic Studies of Hypertension: Mechanism and Risk}

Twin and Sibling Pairs

Applying a classic twin design in the search for traitassociated polymorphisms [37], multiple autonomic phenotypes were measured in twin pairs, permitting estimation of trait heritability and definition of effects of particular genetic variants on "intermediate" (early, pathogenic) phenotypes; simultaneous documentation of heritability and association lends internal validation to the approach. Twins in such allelic/haplotype association studies were self-identified as being of European (white) ancestry, to minimize effects of population stratification.

\section{Blood Pressure Change During Cold Stress: Heritable} "Intermediate" Phenotype of Hypertension

Because systemic hypertension may result from the cumulative effects of transient adverse blood pressure (BP) responses to environmental stress in genetically predisposed individuals [38], we probed the BP response to environmental stress, using cold as a systematic stimulus in a series of predominantly normotensive twin pairs. Stress BP increments were significantly heritable $\left(h^{2}=32 \pm 8 \%, P=0.0003\right.$ for change in diastolic BP $[\mathrm{DBP}] ; h^{2}=23 \pm 9 \%, P=0.0098$ for change in systolic BP [SBP]), as estimated by twin pair variance components [39].

Population Sample With Extreme BP Values: A Statistically Powerful Sample for Association Studies of Hypertension

To pursue the genetic involvement in established essential hypertension, we developed a powerful resource in the form of a population-based sample consisting of individuals with extremely high and low BPs [40]. From a primary care practice sample of over 53,000 people, we ascertained more than 1,000 individuals from the upper and lower 5th percentiles of the population $\mathrm{BP}$ distribution, matched for age, gender, and ethnicity [41]. 


\section{CHGA Genetic Variants and Blood Pressure}

\section{Open Reading Frame}

During resequencing, three variants were discovered in the catestatin region, the most common of which was Gly364Ser, at $\sim 3.1 \%$ of the initial $\sim 360$ chromosomes resequenced [42]. In cultured hippocampal neurons, wild-type human catestatin (SSMKLSFRARAYG ${ }_{364}$ FRGPGPQL) inhibited nicotinic cholinergic-stimulated catecholamine release completely (to $0.3 \pm 2.8 \%$ of control) at $10 \mu \mathrm{mol} / \mathrm{L}$. The same dose of the 364 Ser variant (SSMKLSFRARAYS ${ }_{364}$ FRGPGPQL) inhibited catecholamine release with substantially reduced potency: $35.7 \pm 3.1 \%$ of control secretion persisted [28]. Carriers of 364Ser had diminished renal norepinephrine excretion (by $\sim 26 \% ; P=0.047$ ) and epinephrine excretion (by $\sim 34 \% ; P=0.003$ ); plasma norepinephrine also declined by $\sim 36 \% \quad(P=0.019)$, though plasma epinephrine was unchanged [28]. Gly/Ser heterozygotes displayed increased baroreceptor slope during upward deflections (by $\sim 7 \%$ ) and downward deflections (by $\sim 4 \%$ ), increased cardiac parasympathetic index (by $\sim 2.4$-fold), and decreased cardiac sympathetic index (by $\sim 26 \%$ ). The 364Ser variant was associated with lower diastolic blood pressure in two independent/ confirmatory groups of patients with hypertension (UCSD unrelated, $n=489$; population with blood pressure extreme values, $n=1361$ ); genotype groups differed by $\sim 5-6 \mathrm{~mm} \mathrm{Hg}$, and the polymorphism accounted for $\sim 1.8 \%$ of population diastolic blood pressure variance, although a significant geneby-sex interaction existed, with an predominant effect in men.

\section{Promoter: $C H G A$ Promoter Haplotype Effects on Stress Traits}

Five tightly linked common promoter variants - at positions C$1014 \mathrm{~T}$ (rs9658630), G-988T (rs9658631), G-462A (rs9658634), C-415T (rs9658635), and A-89C (rs7159323)were investigated in a study of 112 phenotyped twin pairs ( $2 n=224$ individuals), and haplotypes were inferred with the HAP algorithm, a method for haplotype resolution from genotype data [43]. The three most common haplotypes, TTGTC (Hap-A, 56.9\%), CGATA (Hap-B, 23.0\%), and TTGCC (Hap-C, 16.5\%), accounted for more than 95\% of all haplotypes. Diploid haplotypic variation in the region affected both the change in DBP $(P=0.0222)$ and the final DBP $(P=0.0167)$ during cold stress [44•]. Homozygosity for Hap-B (CGATA/CGATA) seemed to blunt both the change in DBP and the final DBP, whereas Hap-A/Hap-B heterozygosity (TTGTC/CGATA) was associated with the greatest values of both the change in DBP and the final DBP. The more extreme trait values for heterozygotes (as compared with either homozygote class) suggested the phenomenon of molecular heterosis [45].
CHGA 3'-UTR Variant C+87T (rs7610) and Dichotomous (Hypertension) as well as Continuous (mm Hg) BP Traits

Table $2 \mathrm{a}$ indicates the $\mathrm{C}+87 \mathrm{~T}$ genotype effect in men $(P=$ $0.004)$ and lack of effect in women $(P=0.262)$. To focus on the role of the minor $(\mathrm{T})$ allele, we combined the minor allele homozygote $(\mathrm{T} / \mathrm{T})$ with heterozygote $(\mathrm{C} / \mathrm{T})$ categories, and compared them with $\mathrm{C} / \mathrm{C}$ (major allele) homozygotes. The $\mathrm{C}$ allele displayed a significant effect on blood pressure in men but not in women [46•].

Table $2 b$ shows the effects of $\mathrm{C}+87 \mathrm{~T}$ on SBP and DBP as continuous traits (in $\mathrm{mm} \mathrm{Hg}$ ). Even though these individuals were ascertained from the population by a DBP criterion [41], those who were hypertensive exhibited elevations of both DBP and SBP, and there were significant effects of $\mathrm{C}+87 \mathrm{~T}$ genotype on both $\operatorname{SBP}(P=0.028)$ and DBP $(P=0.037)$. Likewise, there were significant genotype-by-sex interactions on both $\operatorname{SBP}(P=0.015)$ and DBP $(P=0.010)$. In men, BP differences between homozygote $(\mathrm{C} / \mathrm{C}, \mathrm{T} / \mathrm{T})$ classes were substantial: $\sim 12 \mathrm{~mm} \mathrm{Hg}$ for SBP and $\sim 9 \mathrm{~mm} \mathrm{Hg}$ for DBP. By ANOVA (adjusted) $\mathrm{R}^{2}$ in men, $\mathrm{C}+87 \mathrm{~T}$ accounted for $\sim 1.9 \%$ of the population variance in SBP (or $\sim 13.7 \mathrm{~mm} \mathrm{Hg}$ ), and $\sim 1.2 \%$ of the variance for DBP (or $\sim 5.8 \mathrm{~mm} \mathrm{Hg}$ ). In women, $\mathrm{C}+87 \mathrm{~T}$ did not affect either SBP or DBP.

\section{CHGB and Blood Pressure}

CHGB Promoter Variants A-296C and A-261T: Implications for Hypertension

There are two common single nucleotide polymorphisms (SNPs) in the proximal promoter of human CHGB: A-296C (rs236140) and A-261T (rs236141). Promoter haplotype effects upon BP in the population were highly significant (Fig. 2) [47•]. Chromosome-based haplotype analysis on individuals dichotomized into two groups (higher versus lower BP) indicated that individuals with the less common AT or CA haplotypes had a strong tendency to be hypertensive $(\mathrm{OR}=4.898$ for AT haplotype, $P=3.19 \mathrm{E}-11$; $\mathrm{OR}=3.84$ for CA haplotype, $P=3.64 \mathrm{E}-10$ ). People with the more common haplotype $\mathrm{CT}$ had a strong tendency to be normotensive $(\mathrm{OR}=0.637 ; 95 \% \mathrm{CI}, 0.524-0.773 ; P=$ 4.64E-6). The most common haplotype, AA, had no effect on BP status. The overall effect of $C H G B$ haplotypes on $\mathrm{BP}$ status was substantial, as shown on Fig. 2, whether analyzed by chromosome/haplotype (global $\chi^{2}=93.9, P=$ $3.16 \mathrm{E}-20$ ) (left panel) or by diploid haplotype pairs (global $\chi^{2}=75.0, P=4.92 \mathrm{E}-13$ ) (right panel).

We also studied the quantitative trait effects (SBP and DBP in $\mathrm{mm} \mathrm{Hg}$ ) of $C H G B$ haplotypes, and found substantial predictions for both SBP and DBP. With increasing copy number $(0 \rightarrow 1 \rightarrow 2)$ of haplotype AT, SBP increased 
Table 2 CHGA 3'-UTR common variant $\mathrm{C}+87 \mathrm{~T}$ and BP. Effect of the $\mathrm{T}$ allele on blood pressure status and values ${ }^{\mathrm{a}}$

\begin{tabular}{|c|c|c|c|c|}
\hline Sex & Genotype & & & \\
\hline a. $C H G$ & requencies an & mized $)^{b}$ & & \\
\hline & & High BP, $n$ & Low BP, $n$ & $P($ 2-sided $)$ \\
\hline Male & $\mathrm{C} / \mathrm{C}$ & 156 & 107 & \\
\hline & $\mathrm{C} / \mathrm{T}$ or $\mathrm{T} / \mathrm{T}$ & 95 & 112 & 0.004 \\
\hline Female & $\mathrm{C} / \mathrm{C}$ & 112 & 170 & \\
\hline & $\mathrm{C} / \mathrm{T}$ or $\mathrm{T} / \mathrm{T}$ & 119 & 148 & 0.262 \\
\hline b. $C H G$ & and $\mathrm{BP}$ as a $\mathrm{c}$ & & & \\
\hline & & $\mathrm{SBP}(\mathrm{mm} \mathrm{Hg})$ & DBP (mm Hg) & \\
\hline Male & $\mathrm{C} / \mathrm{C}$ & $137.2 \pm 1.6$ & $83.7 \pm 1.3$ & \\
\hline & $\mathrm{C} / \mathrm{T}$ or $\mathrm{T} / \mathrm{T}$ & $131.2 \pm 1.8$ & $77.6 \pm 1.5$ & \\
\hline Male AI & & 0.016 & 0.003 & \\
\hline Female & $\mathrm{C} / \mathrm{C}$ & $123.8 \pm 1.7$ & $71.8 \pm 1.2$ & \\
\hline & $\mathrm{C} / \mathrm{T}$ or $\mathrm{T} / \mathrm{T}$ & $126.9 \pm 1.7$ & $73.8 \pm 1.3$ & \\
\hline Female & & 0.206 & 0.272 & \\
\hline
\end{tabular}

Reprinted from Journal of the American College of Cardiology, Vol. 52. Chen Y, Rao F, Rodriguez-Flores JL, et al.: Naturally occurring human genetic variation in the $3^{\prime}$-untranslated region of the secretory protein chromogranin A is associated with autonomic blood pressure regulation and hypertension in a sex-dependent fashion; Pages 1468-1481, Copyright 2008, with permission from Elsevier

ANOVA analysis of variance; $B P$ blood pressure; $D B P$ diastolic blood pressure; SBP systolic blood pressure; UTR untranslated region

${ }^{a}$ Subjects are grouped by absence $(\mathrm{C} / \mathrm{C})$ or presence $(\mathrm{T} / \mathrm{T}$ or $\mathrm{C} / \mathrm{T})$ of the $\mathrm{T}$ (minor) allele

${ }^{\mathrm{b}}$ Results evaluated by chi-square test

${ }^{\mathrm{c}}$ Allelic association (presence or absence of the T allele) evaluated by univariate ANOVA

by $\sim 29 \mathrm{~mm} \mathrm{Hg}(P=0.0002)$, whereas DBP increased by $\sim 21 \mathrm{~mm} \mathrm{Hg}(P=1.59 \mathrm{E}-5)$, each in additive fashion. With increasing copy number of haplotype CA, SBP increased by $\sim 13 \mathrm{~mm} \mathrm{Hg}(P=0.005)$ and DBP increased by $\sim 12 \mathrm{~mm} \mathrm{Hg}(P=8.49 \mathrm{E}-6)$. With increasing copy number of haplotype CT, SBP decreased by $\sim 11 \mathrm{~mm} \mathrm{Hg}(P=0.0045)$, with a parallel decrease in DBP by $\sim 7 \mathrm{~mm} \mathrm{Hg}(P=0.011)$.
When we adjusted BP values for the effects of antihypertensive medications in treated hypertensives [48], the haplotype effects on SBP/DBP persisted or increased: AT, $P=4.1 \mathrm{E}-5 /$ $P=6.83 \mathrm{E}-6 ; \quad$ CA,$P=0.008 / P=2.04 \mathrm{E}-5 ; \quad \mathrm{CT}, P=0.002 /$ $P=0.008$.

Promoter polymorphisms A-296C/A-261T interacted nonadditively to influence SBP and DBP $(P=1.15 \mathrm{E}-6 / P=$

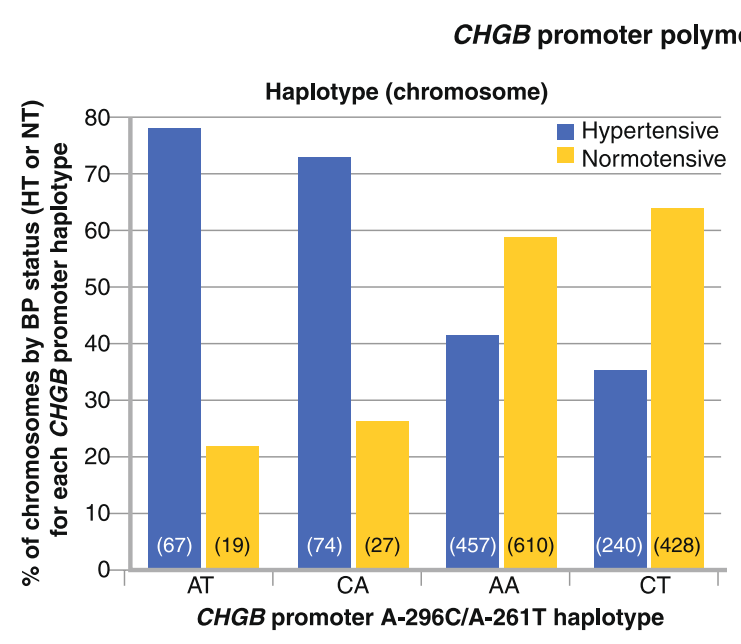

Fig. 2 Prediction of blood pressure (BP) status by $C H G B$ promoter haplotypes in a population (of European ancestry) with BP extremes. Haplotypes (or haplotype pairs) are ordered by rank of association with elevated BP. Left: Haplotype (chromosome) effects. Right: Haplotype pair (diploid haplotype) effects. The two most common

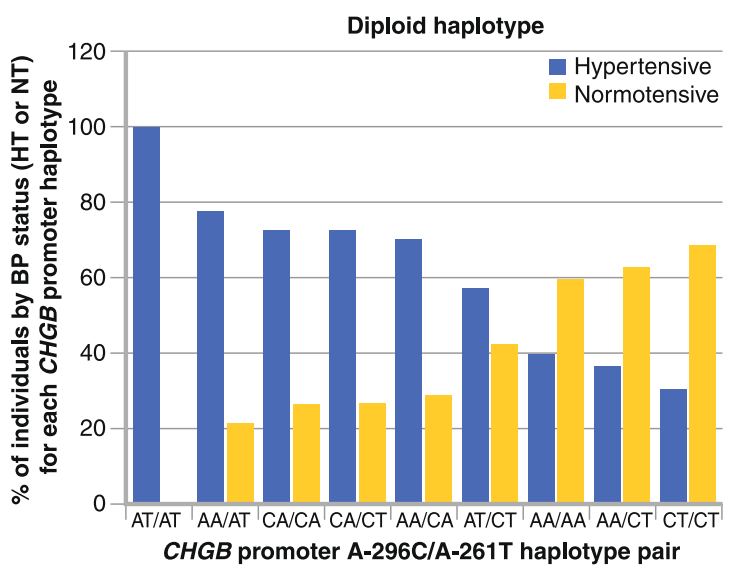

haplotypes (AA and $\mathrm{CT}$ ) are more frequent in individuals with lower $\mathrm{BP}$, whereas the two less common haplotypes (CA and AT) are more frequent in those with higher BP. CHGB - chromogranin B; HThypertensive; NT—normotensive. (Adapted from Zhang et al. [47•]) 
1.07E-10). On a background of A-296C major allele homozygosity (A/A), the A-261T major (A) allele lowered SBP/DBP by $\sim 27 / 22 \mathrm{~mm} \mathrm{Hg}$, whereas on a background of A-296C minor allele homozygosity $(\mathrm{C} / \mathrm{C})$, the A-261T major (A) allele elevated SBP/DBP by $\sim 21 / \sim 18 \mathrm{~mm} \mathrm{Hg}$. When treatment-adjusted BPs [48] were analyzed, the interactions increased or persisted: SBP/DBP, $P=4.41 \mathrm{E}-7 /$ $P=1.08 \mathrm{E}-10$. The A-296C-by-A-261T (SNP-by-SNP) interaction was confirmed on analyses of the dichotomous $\mathrm{BP}$ trait (higher vs lower): $P=3.71 \mathrm{E}-15$.

SBP/DBP values stratified by $C H G B$ promoter haplotypes were, in rank order: $\mathrm{CT}<\mathrm{AA}<\angle \mathrm{CA}<\mathrm{AT}$ (SBP/DBP, $P=$ $6.16 \mathrm{E}-8 / P=5.18 \mathrm{E}-12)$. On ANOVA, $C H G B$ promoter genetic variation accounted for $\sim 2.3 \%$ of SBP variance and $\sim 3.4 \%$ of DBP variance in this primary care population.

Parallel Effects of Human $C H G B$ Promoter Haplotypes In Vivo and in Cells

To probe the functional significance of the two common promoter variants for transcriptional efficiency, we inserted each of the four haplotypes (across A-296C and A-261A) into the luciferase reporter plasmid pGL3-Basic. After transfection into rat chromaffin (PC12) cells, these four haplotypes yielded substantially different luciferase reporter activity. Site-specific analysis of reporter activity showed that both A-296C and A$261 \mathrm{~T}$ have context-dependent actions. The transcriptional strengths of combinations of A-296C and A-261T were $\mathrm{AT}>\mathrm{CT}>\mathrm{AA}>\mathrm{CT} \quad(P=8.46 \mathrm{E}-07) . \quad$ By two-way ANOVA, overall $F=107.2, P=8.46 \mathrm{E}-07$; A-296C, $P=2.09 \mathrm{E}-05$; A261T, $P=0.064$; 296-by-261 interaction, $P=3.07 \mathrm{E}-07$. The four promoter haplotypes displayed the same rank order for effects on BP in vivo and for luciferase reporter activity in chromaffin cells.

\section{"Intermediate" Traits: Association of $C H G B$ Promoter Variants A-296C and A-261T}

In 163 white twin pairs who were predominantly ( $90 \%)$ normotensive, the DBP response to environmental (cold) stress was substantially heritable $\left(h^{2}=32 \pm 8 \%, P=0.0003\right)$ [49]. $C H G B$ promoter individual genotypes A-296C (A $>$ C, $P=0.0237)$ and $\mathrm{A}-261 \mathrm{~T}(\mathrm{~A}>\mathrm{T}, P=0.037)$ predicted the change in DBP in the cold stress test. Because of the modest sample size $(2 n=326$ chromosomes), we observed only five examples of the CA haplotype and one of the AT haplotype, so analyses could only be performed for haplotypes AA and CT. Consistent with basal BP in white BP extremes, the CT haplotype decreased the change in DBP in response to stress, whereas the AA haplotype increased it; thus, the rank order of effects of the haplotypes on $\mathrm{BP}$ was preserved in twins $(\mathrm{AA}>\mathrm{CT})$, though the effects of the more prominent $\mathrm{BP}$-increasing haplotypes (CA, AT) could not be quantified in this predominantly normotensive sample.

CHGB 3'-UTR Common Variant C+84A (rs2821), Isoprostane, and Hypertension

Isoprostane marks oxidative stress, and elevated isoprostane excretion may be involved in cardiovascular target organ damage. Urinary isoprostane excretion was substantially heritable in twin pairs $\left(h^{2}=65.8 \pm 4.3 \%\right)$, and the isoprostane trait aggregated with multiple traits (CHGB, catecholamines, autonomic/baroreceptor sensitivity, and renal function), including several features of the metabolic syndrome (body mass index, insulin resistance, dyslipidemia). Isoprostane excretion also paralleled systemic hypertension. A common variant in the $3^{\prime}$-untranslated region (UTR) of $C H G B$ $(\mathrm{C}+84 \mathrm{~A}$, rs2821) associated with plasma $\mathrm{CHGB}$ and isoprostane excretion. $\mathrm{C}+84 \mathrm{~A}$ disrupted an $\mathrm{A} / \mathrm{U}$-rich mRNA stability element, and in transfected luciferase/3'-UTR plasmids, the $\mathrm{C}+84$ and $+84 \mathrm{~A}$ alleles differed markedly in reporter expression in chromaffin and neuroblastoma cells; site-directed mutagenesis confirmed the importance of this variant within the context of the A/U-rich motif [50॰].

\section{SCG2 and Blood Pressure}

SCG2: A Functional Intronic Variant (G736A, rs1017448, intron-1) Is Associated with Hypertension

At systematic polymorphism discovery at the $S C G 2$ locus, BP associations maximized at variant G736A (rs1017448) in the sole intron; G736A is common (at $~ 30.5 \%$ ) only in African Americans, and the minor allele A seemed to be ancestral [51]. This SNP was investigated in five different cohorts. G736A was associated with blood pressure in African Americans from the University of California, San Diego (UCSD) $(n=383, P=0.0049)$, with the ancestral allele (A) predicting increased risk for hypertension. Significant association was observed in a second cohort of unrelated African Americans from metropolitan San Diego $(n=465)$ for both recessive $(P=0.022)$ and dominant $(P=$ $0.0098)$ models. Taking the two case-control studies as independent observations supports $P \sim 10^{-4}$ for either dominant or recessive models. In African American families from the GenNet network of the NHLBI Family Blood Pressure Program $(n=781)$, analysis shows association of G736A with SBP (FBAT, $P=0.032$ under an additive model) and pulse pressure (FBAT, $P=0.0074$ additive model; $P=0.0076$ for dominant or recessive models), though not with hypertension diagnosis as a dichotomous trait. However, two population samples with extreme BPs - a Nigerian sample $(n=370)$ and a European 
American sample $(n=1,315)$ - showed no significant associations, although the minor allele frequency among European Americans was too low to be sufficiently informative; indeed, we observed no minor allele homozygotes among those 1,315 individuals.

Thus all three African American cohorts showed significant association of the $736 \mathrm{~A}$ allele with elevated blood pressure or hypertension. A meta-analysis was performed of unrelated individuals from all five cohorts, and this analysis showed significant associations in both additive models $(P=0.017)$ and dominant models $(P=$ 0.028), which survived correction for covariates.

Paired-like homeobox transcription factors PHOX2A (ARIX) and PHOX2B were identified by oligonucleotide affinity chromatography and mass spectrometry as potential transactivating factors across the G736A motif; this finding was confirmed by chromatin immunoprecipitation. Each of these transcription factors preferentially binds the risk allele, both in vitro and in vivo [51]. PHOX2A and PHOX2B play roles in autonomic nervous system function; these paralogous genes are expressed in a largely overlapping pattern during development [52], but genetic studies point to unique requirements for each factor in vivo.

\section{Hypertension Consequences: The Kidney}

\section{CHGA and Microalbumin Excretion}

Urinary albumin excretion is a risk factor for adverse cardiovascular events, and even albumin excretion values less than those typically cited as constituting microalbuminuria or incipient nephropathy may signal cardiovascular risk. Urinary excretions of albumin and catecholamines were highly heritable in twin pairs, and their parallel suggests adrenergic mediation of early glomerular permeability alterations [53]; indeed, the heritability of albumin excretion was $\mathrm{h}^{2}=45.2 \pm$ $7.4 \%\left(P=2 \times 10^{-7}\right)$. In the same study, we found that albumin excretion associated with polymorphisms at multiple points in the adrenergic pathway: catecholamine biosynthesis, catabolism, storage/release $(C H G A)$, receptor targets, and postreceptor signal transduction. At $C H G A$, associations were found not only for haplotypes spanning the locus, but also for functional catestatin variant Gly364Ser. Thus interindividual variability in albumin excretion is controlled in substantial part by genetic variation in the adrenergic pathway, including common, naturally occurring polymorphism at the $C H G A$ locus.

\section{CHGA Genetic Variation and Hypertensive Renal Disease}

Among blacks, we studied common genetic variants spanning the CHGA locus in two independent case-control studies of hypertensive end-stage renal disease (ESRD), comprising 58 cases and 150 control subjects. Two haplotypes were significantly more frequent among patients with hypertensive ESRD: 1 ) in the promoter $\left(5^{\prime}\right)$ region, G$462 \mathrm{~A}(\mathrm{rs} 9658634) \rightarrow \mathrm{T}-415 \mathrm{C}(\mathrm{rs} 9658635) \rightarrow \mathrm{C}-89 \mathrm{~A}$ (rs7159323), haplotype ATC (adjusted OR=2.65; $P=$ 0.037); and 2) at the $3^{\prime}$-end, C11825T (3'-UTR, C+87T; rs7610) $\rightarrow$ G12602C (rs875395), haplotype TC (adjusted $\mathrm{OR}=2.73, P=0.0196$ ). Circulating levels of catestatin were lower among those with hypertensive ESRD than among controls, an unexpected finding given that peptide levels are usually elevated in ESRD because of reduced renal elimination [54•].

CHGA Regulates Renal Function by Triggering Weibel-Palade Body Exocytosis From Endothelial Cells

In twin pairs, the CHGA locus is a trans-quantitative trait locus for determination of endothelin-1 (ET-1) secretion, with maximal association in the $C H G A$ promoter region, and the CHGA protein itself triggers ET-1 release from human umbilical vein endothelial cells [55]. Weibel-Palade bodies are the storage granules of endothelial cells. They store and release two principal molecules, von Willebrand factor and P-selectin, and thus play a dual role in hemostasis and inflammation. CHGA led to the release of ET-1 and indeed global Weibel-Palade body exocytosis in cultured human umbilical vein endothelial cells, and the secretory effect mapped onto the amino-terminal (vasostatin) region of the molecule. In addition, CHGA triggered secretion of ET1 from glomerular capillary endothelial cells and TGF- $\beta 1$ from co-cultured mesangial cells. In humans, plasma CHGA correlated positively with ET-1 and negatively with the glomerular filtration rate (GFR). GFR was highly heritable in twin pairs, and common promoter haplotypes of $C H G A$ predicted the GFR trait value. In patients with progressive hypertensive renal disease, a $C H G A$ haplotype predicted the rate of GFR decline. Thus CHGA seems to act through the glomerular endothelium to regulate renal function as well as progressive renal disease $[56 \bullet]$.

\section{An Experimental Animal Model: Elevated BP After Targeted Chromogranin Gene Ablation in the Mouse}

Chga-/- (knockout) mice displayed extreme phenotypic changes, including: decreased chromaffin granule size and number; elevated BP; loss of diurnal BP variation; increased left ventricular mass and cavity dimensions; decreased adrenal catecholamine, neuropeptide $\mathrm{Y}$ (Npy), and ATP contents; increased catecholamine/ATP ratio in the chromaffin granule; and increased plasma catecholamine and Npy 
levels. Rescue of elevated BP to normalcy was achieved by either exogenous catestatin replacement or humanization of Chga-/- mice. Loss of the physiological "brake" catestatin in Chga-/- mice, coupled with dysregulation of transmitter storage and release, may act in concert to alter autonomic control of the circulation in vivo, eventuating in hypertension [3]. The syndrome could be "rescued" by BAC (bacterial artificial chromosome) haplotype replacement of the human CHGA gene into the mouse knockout strain genome [3, 57•].

The Chga-/- mouse displays widespread derangements in autonomic function, both sympathetic and parasympathetic [58•], including diminished baroreceptor sensitivity to both high-pressure and low-pressure stimuli and diminished heart rate variability [59.]; each of these can be rescued by replacement of the catestatin (catecholamine release inhibitory) fragment of CHGA.

Later studies indicated that the relationship of the Chga gene to BP may be even more complex, with a biphasic (or U-shaped) dose/response curve for Chga gene copy number and basal BP [57•] and parallel changes in adrenal catecholamine storage and release. In this transgenic model, titration of $C H G A$ gene copy number was enabled by BAC haplotype replacement and targeted breeding.

Finally, loss of pancreastatin in the Chga-/- mouse has predictable metabolic consequences, including heightened sensitivity of glucose disposal to endogenous or exogenous insulin $[60 \bullet, 61 \bullet]$. The insulin-hypersensitive state is reversed by replacement of the dysglycemic pancreastatin fragment of CHGA [61•].

Similarly, Chgb-/- (knockout) mice display substantially higher SBP and DBP (by $\sim 20 / \sim 18 \mathrm{~mm} \mathrm{Hg}$ ) than $C h g b+/+$ mice [62•]. These mice have $36 \%$ less adrenomedullary epinephrine when compared to wild-type mice, whereas the norepinephrine content is similar. This decrease is not due to a lower frequency of exocytotic events, but rather to less secretion per quantum ( $230 \%)$ measured by amperometry; amperometric spikes exhibit a slower ascending phase but a normal decaying phase [63]. Chgb-/- animals display some, but not all, hallmarks of human type 2 diabetes. Stimulated secretion of insulin, glucagon, and somatostatin is reduced in Chgb-/- islets, in parallel with somewhat impaired glucose clearance and reduced insulin release, but normal insulin sensitivity in vivo [64].

\section{Conclusions}

The major soluble core proteins in catecholamine storage vesicles (CHGA, CHGB, and SCG2) play catalytic roles in vesiculogenesis. Common genetic variants in these proteins, especially $C H G A 3^{\prime}$-UTR C $+87 \mathrm{~T}$ (rs7610), $C H G B$ promoter A-296C (rs236140) and A-261T (rs236141), and SCG2 intronic variant G736A (rs1017448), seem to exert functional and interactive effects, first on early, "intermediate," or pathogenic phenotypes and finally on basal/ resting BP in the population, leading to risk of hypertension. SNP-by-SNP interactions, either local (haplotypes) or distant, may also play roles in the progression of BP. These results point to new molecular strategies for probing autonomic control of the circulation and ultimately the susceptibility to and pathogenesis of cardiovascular disease states such as hypertension.

Acknowledgment These authors have received support from the US Department of Veterans Affairs and the National Institutes of Health (HL58120, RR00827, MD000220).

Disclosure No conflicts of interest relevant to this article were reported.

Open Access This article is distributed under the terms of the Creative Commons Attribution Noncommercial License which permits any noncommercial use, distribution, and reproduction in any medium, provided the original author(s) and source are credited.

\section{References}

Papers of particular interest, published recently, have been highlighted as:

- Of importance

1. Mahapatra NR, Mahata M, Mahata SK, O'Connor DT: The chromogranin A fragment catestatin: specificity, potency and mechanism to inhibit exocytotic secretion of multiple catecholamine storage vesicle co-transmitters. J Hypertens 2006, 24:895-904.

2. Videen JS, Mezger MS, Chang YM, O'Connor DT: Calcium and catecholamine interactions with adrenal chromogranins. Comparison of driving forces in binding and aggregation. $\mathrm{J}$ Biol Chem 1992, 267:3066-3073.

3. Mahapatra NR, O'Connor DT, Vaingankar SM, et al.: Hypertension from targeted ablation of chromogranin A can be rescued by the human ortholog. J Clin Invest 2005, 115:1942-1952.

4. Yoo SH, So SH, Huh YH, Park HY: Inositol 1,4,5-trisphosphate receptor $/ \mathrm{Ca}(2+)$ channel modulatory role of chromogranins $\mathrm{A}$ and B. Ann N Y Acad Sci 2002, 971:300-310.

5. Mahata SK, Kozak CA, Szpirer J, et al.: Dispersion of chromogranin/secretogranin secretory protein family loci in mammalian genomes. Genomics 1996, 33:135-139.

6. Huh YH, Jeon SH, Yoo SH: Chromogranin B-induced secretory granule biogenesis: comparison with the similar role of chromogranin A. J Biol Chem 2003, 278:40581-40589.

7. Kim T, Tao-Cheng JH, Eiden LE, Loh YP: Chromogranin A, an "on/off" switch controlling dense-core secretory granule biogenesis. Cell 2001, 106:499-509.

8. Beuret N, Stettler H, Renold A, et al.: Expression of regulated secretory proteins is sufficient to generate granule-like structures in constitutively secreting cells. J Biol Chem 2004, 279:20242-20249.

9. Taupenot L, Harper KL, O'Connor DT: The chromograninsecretogranin family. N Engl J Med 2003, 348:1134-1149.

10. Tatemoto K, Efendic S, Mutt V, et al.: Pancreastatin, a novel pancreatic peptide that inhibits insulin secretion. Nature 1986, 324:476-478. 
11. Zhang K, Rao F, Wen G, et al.: Catecholamine storage vesicles and the metabolic syndrome: The role of the chromogranin A fragment pancreastatin. Diabetes Obes Metab 2006, 8:621-633.

12. Strub JM, Hubert P, Nullans G, et al.: Antibacterial activity of secretolytin, a chromogranin B-derived peptide (614-626), is correlated with peptide structure. FEBS Lett 1996, 379:273-278.

13. Aardal S, Helle KB, Elsayed S, et al.: Vasostatins, comprising the $\mathrm{N}$-terminal domain of chromogranin A, suppress tension in isolated human blood vessel segments. J Neuroendocrinol 1993, 5:405-412.

14. Mahata SK, O'Connor DT, Mahata M, et al.: Novel autocrine feedback control of catecholamine release. A discrete chromogranin a fragment is a noncompetitive nicotinic cholinergic antagonist. $\mathrm{J}$ Clin Invest 1997, 100:1623-1633.

15. Mahata SK, Mahata M, Parmer RJ, O'Connor DT: Desensitization of catecholamine release. The novel catecholamine releaseinhibitory peptide catestatin (chromogranin a344-364) acts at the receptor to prevent nicotinic cholinergic tolerance. J Biol Chem 1999, 274:2920-2928.

16. Benjannet S, Leduc R, Adrouche N, et al.: Chromogranin B (secretogranin I), a putative precursor of two novel pituitary peptides through processing at paired basic residues. FEBS Lett 1987, 224:142-148.

17. Benjannet S, Leduc R, Lazure C, et al.: GAWK, a novel human pituitary polypeptide: isolation, immunocytochemical localization and complete amino acid sequence. Biochem Biophys Res Commun 1985, 126:602-609.

18. Kroesen S, Marksteiner J, Leitner B, et al.: Rat brain: distribution of immunoreactivity of PE-11, a peptide derived from chromogranin B. Eur J Neurosci 1996, 8:2679-2689.

19. Metz-Boutigue MH, Goumon Y, Lugardon K, et al.: Antibacterial peptides are present in chromaffin cell secretory granules. Cell Mol Neurobiol 1998, 18:249-266.

20. Strub JM, Garcia-Sablone P, Lonning K, et al.: Processing of chromogranin $\mathrm{B}$ in bovine adrenal medulla. Identification of secretolytin, the endogenous C-terminal fragment of residues 614626 with antibacterial activity. Eur J Biochem 1995, 229:356-368.

21. Wiedermann CJ: Secretoneurin: a functional neuropeptide in health and disease. Peptides 2000, 21:1289-1298.

22. Takiyyuddin MA, Baron AD, Cervenka JH, et al.: Suppression of chromogranin-A release from neuroendocrine sources in man: pharmacological studies. J Clin Endocrinol Metab 1991, 72:616-622.

23. Dimsdale JE, O'Connor DT, Ziegler M, Mills P: Chromogranin A correlates with norepinephrine release rate. Life Sci 1992, 51:519-525.

24. Takiyyuddin MA, Parmer RJ, Kailasam MT, et al.: Chromogranin $\mathrm{A}$ in human hypertension. Influence of heredity. Hypertension 1995, 26:213-220.

25. Sanchez-Margalet V, Valle M, Lobon JA, et al.: Plasma pancreastatin-like immunoreactivity correlates with plasma norepinephrine levels in essential hypertension. Neuropeptides 1995, 29:97-101.

26. Sanchez-Margalet V, Valle M, Lobon JA, et al.: Increased plasma pancreastatin-like immunoreactivity levels in non-obese patients with essential hypertension. J Hypertens 1995, 13:251258.

27. O'Connor DT, Kailasam MT, Kennedy BP, et al.: Early decline in the catecholamine release-inhibitory peptide catestatin in humans at genetic risk of hypertension. J Hypertens 2002, 20:1335-1345.

28. Rao F, Wen G, Gayen JR, et al.: Catecholamine release-inhibitory peptide catestatin (chromogranin A(352-372)): naturally occurring amino acid variant Gly364Ser causes profound changes in human autonomic activity and alters risk for hypertension. Circulation 2007, 115:2271-2281.

29. O'Connor DT, Takiyyuddin MA, Printz MP, et al.: Catecholamine storage vesicle protein expression in genetic hypertension. Blood Press 1999, 8:285-295.
30. Schober M, Howe PR, Sperk G, et al.: An increased pool of secretory hormones and peptides in adrenal medulla of strokeprone spontaneously hypertensive rats. Hypertension 1989, 13:469-474.

31. Fries RS, Mahboubi P, Mahapatra NR, et al.: Neuroendocrine transcriptome in genetic hypertension: multiple changes in diverse adrenal physiological systems. Hypertension 2004, 43:1301-1311

32. Greenwood TA, Cadman PE, Stridsberg M, et al.: Genome-wide linkage analysis of chromogranin $\mathrm{B}$ expression in the $\mathrm{CEPH}$ pedigrees: implications for exocytotic sympathochromaffin secretion in humans. Physiol Genomics 2004, 18:119-127.

33. Greenwood TA, Rao F, Stridsberg M, et al.: Pleiotropic effects of novel trans-acting loci influencing human sympathochromaffin secretion. Physiol Genomics 2006, 25:470-479.

34. Takiyyuddin MA, De Nicola L, Gabbai FB, et al.: Catecholamine secretory vesicles. Augmented chromogranins and amines in secondary hypertension. Hypertension 1993, 21:674-679

35. Lillie EO, O'Connor DT: Early phenotypic changes in hypertension: a role for the autonomic nervous system and heredity. Hypertension 2006, 47:331-333.

36. Kirchmair R, Gander R, Egger M, et al.: The neuropeptide secretoneurin acts as a direct angiogenic cytokine in vitro and in vivo. Circulation 2004, 109:777-783.

37. Boomsma D, Busjahn A, Peltonen L: Classical twin studies and beyond. Nat Rev Genet 2002, 3:872-882.

38. Folkow B: Physiological aspects of primary hypertension. Physiol Rev 1982, 62:347-504.

39. Zhang L, Rao F, Wessel J, et al.: Functional allelic heterogeneity and pleiotropy of a repeat polymorphism in tyrosine hydroxylase: prediction of catecholamines and response to stress in twins. Physiol Genomics 2004, 19:277-291.

40. Schork NJ, Nath SK, Fallin D, Chakravarti A: Linkage disequilibrium analysis of biallelic DNA markers, human quantitative trait loci, and threshold-defined case and control subjects. Am J Hum Genet 2000, 67:1208-1218

41. Rana BK, Insel PA, Payne SH, et al.: Population-based sample reveals gene-gender interactions in blood pressure in White Americans. Hypertension 2007, 49:96-106.

42. Wen G, Mahata SK, Cadman P, et al.: Both rare and common polymorphisms contribute functional variation at $\mathrm{CHGA}$, a regulator of catecholamine physiology. Am J Hum Genet 2004, 74:197-207.

43. Halperin E, Eskin E: Haplotype reconstruction from genotype data using Imperfect Phylogeny. Bioinformatics 2004, 20:1842-1849.

44. - Chen Y, Rao F, Rodriguez-Flores JL, et al.: Common genetic variants in the chromogranin A promoter alter autonomic activity and blood pressure. Kidney Int 2008, 74:115-125. The authors uncovered a transcriptional basis whereby common variants and haplotypes in the CHGA promoter may be predictive of heritable changes in autonomic activity and $B P$.

45. Comings DE, MacMurray JP: Molecular heterosis: a review. Mol Genet Metab 2000, 71:19-31.

46. - Chen Y, Rao F, Rodriguez-Flores JL, et al.: Naturally occurring human genetic variation in the 3 '-untranslated region of the secretory protein chromogranin A is associated with autonomic blood pressure regulation and hypertension in a sex-dependent fashion. J Am Coll Cardiol 2008, 52:1468-1481. Here the authors defined the functional activity of common variant $C+87 T$ in the 3'UTR of the CHGA mRNA, which has been associated with both $B P$ elevation in the population and risk for hypertensive (nondiabetic) renal disease.

47. - Zhang K, Rao F, Wang L, et al.: Common functional genetic variants in catecholamine storage vesicle protein promoter motifs interact to trigger systemic hypertension. J Am Coll Cardiol 2010, 55:1463-1475. In this report, common variants and haplotypes of the CHGB promoter were functionally defined in transcriptional 
assays; such variants were strongly predictive of autonomic activity and $B P$ in vivo.

48. Cui JS, Hopper JL, Harrap SB: Antihypertensive treatments obscure familial contributions to blood pressure variation. Hypertension 2003, 41:207-210.

49. Seasholtz TM, Wessel J, Rao F, et al.: Rho kinase polymorphism influences blood pressure and systemic vascular resistance in human twins: role of heredity. Hypertension 2006, 47:937-947.

50. - Rao F, Zhang K, Khandrika S, et al.: Isoprostane, an "intermediate phenotype" for oxidative stress heritability, risk trait associations, and the influence of chromogranin B polymorphism. J Am Coll Cardiol 2010, 56:1338-1350. A common variant $(C+84 A)$ in the 3 '-UTR of the CHGB mRNA was associated with an "oxidative stress" phenotype: isoprostane excretion. The variant acted by disrupting an A/U-rich $m R N A$ stability element.

51. Wen G, Wessel J, Zhou W, et al.: An ancestral variant of Secretogranin II confers regulation by PHOX2 transcription factors and association with hypertension. Hum Mol Genet 2007, 16:1752-1764.

52. Pattyn A, Morin X, Cremer H, et al.: Expression and interactions of the two closely related homeobox genes Phox $2 \mathrm{a}$ and Phox $2 \mathrm{~b}$ during neurogenesis. Development 1997, 124:4065-4075.

53. Rao F, Wessel J, Wen G, et al.: Renal albumin excretion: twin studies identify influences of heredity, environment, and adrenergic pathway polymorphism. Hypertension 2007, 49:1015-1031.

54. - Salem RM, Cadman PE, Chen Y, et al.: Chromogranin A polymorphisms are associated with hypertensive renal disease. J Am Soc Nephrol 2008, 19:600-614. This was the initial report on the effect of CHGA polymorphisms on susceptibility to hypertensive renal disease in African Americans. The functional variant was at position $C+87 T$ in the CHGA mRNA.

55. Lillie EO, Mahata M, Khandrika S, et al.: Heredity of endothelin secretion: human twin studies reveal the influence of polymorphism at the chromogranin A locus, a novel determinant of endothelial function. Circulation 2007, 115:2282-2291.

56. - Chen Y, Mahata M, Rao F, et al.: Chromogranin A regulates renal function by triggering Weibel-Palade body exocytosis. J Am Soc Nephrol 2009, 20:1623-1632. The authors found that CHGA influenced renal function by triggering generalized exocytosis from Weibel-Palade bodies, the hormone storage granules in endothelial cells.

57. • Vaingankar SM, Li Y, Biswas N, et al.: Effects of chromogranin A deficiency and excess in vivo: biphasic blood pressure and catecholamine responses. J Hypertens 2010, 28:817-825. CHGA expression displays paradoxic relationships with $B P$ : though human essential hypertension is associated with elevated plasma CHGA, targeted ablation of the Chga locus in the mouse also results in hypertension. In this study, the authors used gene targeting and replacement to systematically vary the copy number of CHGA from 0 to 4 copies per diploid genome, and the results established biphasic effects of CHGA copy number on catecholamine storage and release as well as on $B P$.

58. - Gayen JR, Gu Y, O’Connor DT, Mahata SK: Global disturbances in autonomic function yield cardiovascular instability and hypertension in the chromogranin a null mouse. Endocrinology 2009, 150:5027-5035. Targeted ablation of the Chga locus results in systemic hypertension in the mouse; this study defined widespread effects of such genetic deletion upon both the sympathetic and parasympathetic branches of the autonomic nervous system.

59. • Dev NB, Gayen JR, O'Connor DT, Mahata SK: Chromogranin a and the autonomic system: decomposition of heart rate variability and rescue by its catestatin fragment. Endocrinology 2010, 151:2760-2768. Adjustment of heart rate upwards or downwards in response to endogenous and exogenous cues (heart rate variability, HRV) reflects a healthy autonomic system. After targeted ablation of the Chga gene in the mouse, HRV declines; the trait can be "rescued" by replacement of the catestatin fragment of CHGA.

60. - Gayen JR, Saberi M, Schenk S, et al.: A novel pathway of insulin sensitivity in chromogranin A null mice: a crucial role for pancreastatin in glucose homeostasis. J Biol Chem 2009, 284:28498-28509. In the mouse, knockout of the Chga gene (Chga-/-genotype) results in a peculiar combination of elevated blood pressure coupled with enhanced insulin sensitivity, especially in the liver. Chga encodes a dysglycemic fragment, named "pancreastatin"; administration of this peptide to Chga-/- mice reversed the insulin sensitivity of the knockout state.

61. • Friese RS, Gayen JR, Mahapatra NR, et al.: Global metabolic consequences of the chromogranin A-null model of hypertension: transcriptomic detection, pathway identification, and experimental verification. Physiol Genomics 2010, 40:195-207. Here the authors profiled the transcriptome in mice after targeted ablation of the Chga locus, and thereby identified global patterns of $m R N A$ expression subserving enhanced insulin sensitivity in this animal model.

62. - Zhang K, Rao F, Rana BK, et al.: Autonomic function in hypertension: Role of genetic variation at the catecholamine storage vesicle protein chromogranin B (CHGB). Circ Cardiovasc Genet 2009, 2:46-56. Here the authors defined commonly occurring, natural genetic variation across the human $C H G B$ locus, including promoter variants predicting elevated BP. In a complementary study, targeted ablation of the mouse Chgb locus resulted in systemic hypertension.

63. Diaz-Vera J, Morales YG, Hernandez-Fernaud JR, et al.: Chromogranin $\mathrm{B}$ gene ablation reduces the catecholamine cargo and decelerates exocytosis in chromaffin secretory vesicles. J Neurosci 2010, 30:950-957.

64. Obermuller S, Calegari F, King A, et al.: Defective secretion of islet hormones in chromogranin-B deficient mice. PLoS One 2010, 5:e8936. 